\title{
Outcomes of sphenopalatine and internal maxillary artery ligation inside the pterygopalatine fossa for posterior epistaxis*
}

\author{
Kristina Piastro', Robert Scagnelli², Neil Gildener-Leapman', \\ Carlos D. Pinheiro-Neto'
}

Rhinology 56; 2: 144-148, 2018

https://doi.org/10.4193/Rhin17.212
' Division of Otolaryngology / Head and Neck Surgery, Department of Surgery, Albany Medical Center, Albany, New York, NY, USA 2 Albany Medical College, Albany, New York, NY, USA
*Received for publication: October 17, 2017

Accepted: November 29, 2017

\begin{abstract}
Objective: Analysis of the efficacy of sphenopalatine artery (SPA) and internal maxillary artery (IMAX) ligation within the pterygopalatine fossa to control posterior epistaxis.
\end{abstract}

Methods: Demographic and clinical data were collected in sixty-two consecutive patients who had SPA/IMAX ligation surgery. Clinical outcomes such as re-bleed rates and complications were acquired.

Results: A total of 62 patients were studied. Thirty-eight percent of patients had previously undergone silver nitrate nasal cautery for epistaxis. Nine patients had undergone previous attempt of SPA procedure or embolization in other services. Two patients returned to the operating room for anterior ethmoid ligation. There was one mortality within 30 days of surgery. Follow up ranged from 3 months to 56 months (median= 28 months).

Conclusions: Dual SPA and IMAX ligation is effective in the control of difficult epistaxis cases, even in those patients with prior surgical intervention.

Key words: epistaxis, sphenopalatine artery ligation, nosebleeds, pterygopalatine fossa

\section{Introduction}

Epistaxis is a common complaint. Approximately $60 \%$ of people will experience an episode in their lifetimes. Epistaxis occurs in a bimodal distribution, affecting predominantly males that are $<10$ years or $>50$ years old. Epistaxis has been identified more commonly in patients with other cardiovascular comorbidities. Roughly two-thirds of these cases will be spontaneous and most will self-resolve. However, approximately $6 \%$ of patients will require either medical or surgical interventions. An even smaller percentage, $0.2 \%{ }^{(1)}$, will require hospitalization for control of hemorrhage.

Location of nasal hemorrhage is divided into anterior and posterior sources. The majority of bleeding is anterior in location and can be treated conservatively, either with cautery, anterior nasal packing or simple application of dorsal nasal pressure. Posterior bleeds occur in approximately 5 to $10 \%$ of cases. Nasal packing has been shown to fail to control posterior epistaxis in up to $48 \%$ of patients ${ }^{(2)}$. When conservative measures fail more invasive means to obtain control of bleeding may be required. The bleeding site of a posterior epistaxis is located at Woodruff's plexus, over the posterior middle turbinate or along the posterior superior aspect of the septum or lateral nasal wall. Branches of the sphenopalatine artery (SPA) are responsible for such epistaxis. SPA ligation is the most common surgical procedure performed for posterior epistaxis that is refractory to nasal packing ${ }^{(3)}$. Previously a CaldwellLuc approach was preferred, however with the advancement of endoscopic techniques, authors have been able to demonstrate decreased complication rate and a shorter length of hospital stay ${ }^{(4)}$.

The terminal portion of the IMAX is located within the pterygopalatine fossa. It gives off several branches in that location, including a terminal branch that passes through the sphe- 
nopalatine foramen along the lateral nasal wall and it is named sphenopalatine artery. This artery can arborize into ten terminal branches, and it is therefore important to either identify and ligate all branches or have the possibility of persistent bleeding ${ }^{(5,6)}$. In order to avoid the often-difficult process of identification of the branching pattern of the SPA, especially in actively hemorrhaging patients, we propose ligation of the artery laterally within the pterygopalatine fossa.

Our objective is to analyze the success rates of SPA/IMAX ligation within the pterygopalatine fossa to control severe posterior epistaxis.

\section{Materials and methods}

A retrospective analysis of all consecutive patients undergoing SPA/IMAX ligation between January 2013 and June 2017 performed by the senior author (CPN). Our Institutional Review Board approved this retrospective chart review. Standard demographic data including: age, sex, anticoagulant use, severity of bleed, and prior history of SPA ligation, nasal cautery, and/or embolization procedures.

Selection criteria and indications for procedure:

1. History of severe posterior epistaxis requiring packing.

2. Failure of standard in office techniques as well as conservative measures such as humidification.

3. Failure of anterior-posterior nasal packing.

Clinical efficacy and outcomes data were gleaned from postoperative patient chart review. Data was collected with attention for postoperative complications; including dry eye and V2 hypoesthesia, blood transfusion, return to the operating room for epistaxis, other management of recurrent epistaxis, and basic laboratory data.

Long-term follow-up data was gathered by chart review as well as phone interview. The total follow up time was defined as the time from surgery to the date of the last clinic visit or phone interview. In total 44 out of 62 patients were reached via telephone for supplementary interview. The remaining 18 patients' follow up was based solely on information from the patient medical record. Six specific questions were assessed in all patients: whether there was an improvement in frequency and severity of nosebleeds; if any nosebleeds happened since the surgery; if any severe nosebleeds happened requiring urgent medical evaluation; if any nasal packing was placed after surgery; if any local cauterization was needed since surgery; and if any revision SPA ligation or embolization were required.

\section{Surgical technique}

After the patient was placed under general anesthesia with orotracheal intubation, a $0^{\circ}$ endoscope was used to inspect both nasal cavities. In case of urgent surgery, the nasal packing was removed only after all instrumentation was ready. Pledgets soaked in oxymetazoline were placed in the nasal cavity. Then

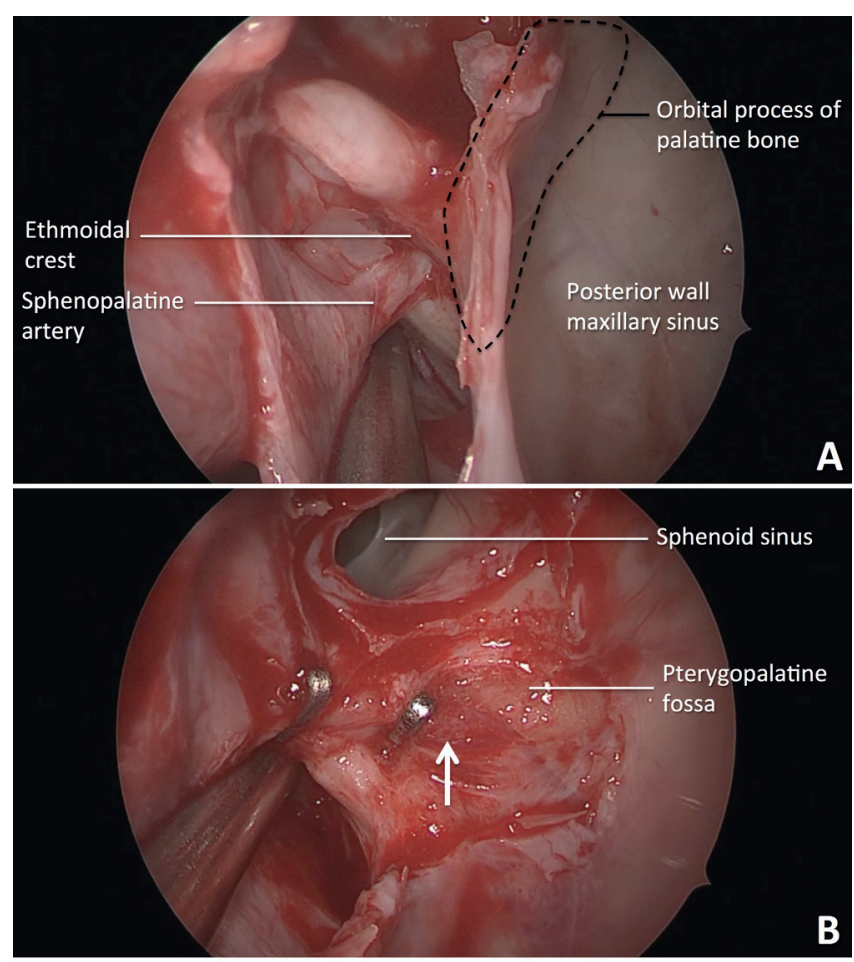

Figure 1. (A) Orbital process of the palatine bone (dashed line). (B) The first hemoclip ligates the sphenopalatine artery. The second and third (arrow) hemoclips are placed inside the pterygopalatine fossa to ligate the internal maxillary artery.

careful medialization of the middle turbinate was performed followed by uncinectomy and wide maxillary antrostomy. Partial anterior and posterior ethmoidectomy were subsequently completed, opening the inferior portion of the posterior ethmoid and leaving the superior portion of the ethmoid intact. This is done to have a better control of the orbital process of the palatine bone during the opening of the pterygopalatine fossa. This partial ethmoidectomy also opens a larger working space for control of epistaxis. The mucosa over the perpendicular plate of the palatine bone (posterior to the posterior fontanelle) was elevated. The dissection was progressed posteriorly until identification of the ethmoidal crest and the sphenopalatine foramen. The SPA was identified. At that point, a $2 \mathrm{~mm}$ Kerrison rongeur was used to remove the orbital process of the palatine bone through the sphenopalatine foramen. That step is performed carefully to avoid injury to the artery. Part of the posterior wall of the maxillary sinus was also removed. The periosteum of the pterygopalatine fossa was exposed and carefully opened with a Cottle dissector to expose the most proximal aspect of the artery. Three titanium-made hemoclips were used for the ligation. Karl Storz ${ }^{\circledR}$ (Tuttlingen, Germany) hemoclip applier was used. One hemoclip was placed to ligate the SPA between the palatine bone and the mucosal flap and the other two clips were placed to ligate the IMAX inside the pterygopalatine fossa. Finally oxidized cellulose 
Table 1. Patient demographics, pre-operative clinical and laboratory tests.

\begin{tabular}{|ll|}
\hline & All Patients \\
\hline Age & $\mathrm{N}=62$ \\
\hline Sex & $18-96$ yo (median $=60.5)$ \\
\hline BMI & $65 \%$ male \\
\hline Estimated Blood Loss (mL) & $29.2 \pm 5.6$ \\
\hline Packings prior to surgery & $161.7 \pm 169.4$ \\
\hline Previous cauterization & $1.82 \pm 2.5$ \\
\hline Previous SPA ligation/Emboli- & $23 / 62(37,1 \%)$ \\
\hline zation & $9 / 62(14,5 \%)$ \\
\hline Hypertension & $30 / 62(48,4 \%)$ \\
\hline Anticoagulant Use & $42 / 62(67,7 \%)$ \\
\hline Pre-op Hemoglobin & $10.6 \pm 2.77$ \\
\hline Pre-op Hematocrit & $32.2 \pm 8.04$ \\
\hline Pre-op Platelets & $225 \pm 63$ \\
\hline
\end{tabular}

$\mathrm{BMI}=$ Body mass index; SPA = Sphenopalatine artery; Pre-op = Preoperative

was used to cover the pterygopalatine fossa and clips (Figure 1).

\section{Results}

Between January 2013 and June 2017 a total of 62 patients underwent SPA/IMAX ligation, with 12 patients undergoing concomitant septoplasty. The demographic data as follows is summarized in Table 1. Forty-one patients (66\%) underwent the procedure acutely as an inpatient and 21 patients (34\%) had planned outpatient surgery after discharge from the hospital. Forty patients (65\%) were male. Patient age varied from 18 to 96 years old, with an average age of 61 years (median $=60.5$ ). Anticoagulant and/or antiplatelet use was present in $67.7 \%$ of the patient population, and hypertension was identified on patient presentation in $48.4 \%$.

Recorded emergent visits for epistaxis were available for 58 patients with an average of $2.55 \pm 2.71$ visits. The number of previous interventions with anterior-posterior nasal packing per patient was $1.82 \pm 2.53$ packings.

Preoperative lab work was available for 53 patients. Preoperative hemoglobin was $10.6 \pm 2.77$, preoperative hematocrit was 32.2 \pm 8.04 , and preoperative platelets were $225 \pm 63$.

Thirty-seven percent of patients had previously undergone silver nitrate nasal cautery for epistaxis. $14.5 \%$ of patients had undergone previous attempt at SPA procedure or embolization in other services. Intra-operative estimated blood loss was available for 50 of the patients and was highly variable (161.7
$\mathrm{mL} \pm 169.4)$.

The clinical follow-up ranged from 3 months to 56 months (Median follow up is 28 months). Seven patients (11.3\%) reported at least one episode of mild nosebleeds after surgery. All patients who had an episode of anterior epistaxis after surgery had a history of anticoagulant and/or antiplatelet use. Two of those patients (3.2\%) who had mild epistaxis required placement of anterior absorbable packing. One patient reported office cauterization with silver nitrate 6 months after surgery. Two patients required anterior ethmoid artery ligation in a separate setting for recurrent anterior epistaxis. None of the 62 patients required revision SPA/IMAX ligation. One patient presented to the emergency room within 30 days of surgery with bilateral posterior nasal bleeding after having left side SPA/IMAX ligation. This patient had bilateral anterior-posterior packing placed with a subsequent cardiac arrest and death. There were no reports of V2 hypoesthesia or dry eye in our patient population.

\section{Discussion}

The management of epistaxis has evolved in recent years. Endoscopic approaches have greatly added to the armamentarium of control of nosebleeds. Endoscopic SPA ligation has become the mainstay of treatment for posterior epistaxis that is refractory to packing or medical management ${ }^{(7)}$. The SPA anatomy is varied and the surgeon must be aware of its branching pattern ${ }^{(5)} .14 .5 \%$ of our patients had previously undergone SPA cautery, ligation or embolization procedures performed in other institutions. The published rate of failure for SPA cauterization or ligation varies from $2 \%$ to $13 \%{ }^{(8-11)}$. Anatomic variations of the SPA branches and foramen location may contribute to inadequate epistaxis control. We observed overall $95.2 \%$ control of epistaxis with ligation of the SPA associated with ligation of the IMAX inside the pterygopalatine fossa. Importantly there were no complications specific to the approach such as dry eye or V2 hypoesthesia.

Bleeding from the anterior or posterior ethmoidal arteries can also be a cause of epistaxis after SPA ligation ${ }^{(9,12)}$. From our 9 patients (14.5\%) who had undergone prior surgical procedures to address the SPA in other services, all of them presented with posterior bleeding, which indicates a local failure to control the SPA. None of them presented with bleeding from the anterior ethmoidal artery area. For our SPA-IMAX ligation series two patients presented with severe anterior bleeding months after initial surgery, these patients were revised with anterior ethmoid artery ligation. During these anterior ethmoid artery ligation surgeries, no bleeding was present from the SPA area and no intervention was performed in that region.

One patient with severe comorbidities required bilateral anterior-posterior packing within 30 days after having left sided SPA-IMAX ligation. He had a cardiac arrest immediately after the packing was placed and subsequent death. It was not possible 
to identify the exact location of the bleeding since the patient was having bilateral hemorrhage.

The age range in this population was 18-96 years old. A posterior bleed in a patient as young as 18 is rare. This patient initially presented with a posterior bleed on post-operative day eight of a functional endoscopic sinus surgery.

All patients had at least two severe nosebleeds prior to surgery with need for anterior-posterior packing or one severe bleed and failure of the anterior-posterior packing. The nasal packing average prior to the surgery in our patient population was 1.8 packs. Pre-operative hemoglobin levels $(10.6 \pm 2.77)$ and hematocrit levels $(32.2 \pm 8.04)$ were both below normal in our patient population, likely a result of the recurrent posterior bleeding prior to surgery.

Seven patients (11.3\%) reported at least one episode of mild nosebleeds after surgery on clinical follow-up. Anteroseptal location was evident on exam and this was treated with simple silver nitrate anterior cautery or absorbable packing placed in contact to the anterior septum with resolution of symptoms.

The seven patients who experienced anterior nosebleeds were on an anticoagulant or antiplatelet medicine. It is important to be vigilant for anterior bleeding as other arteries like the facial artery and anterior ethmoidal artery have an important role in blood supply to the anterior septum ${ }^{(13)}$. Importantly, in our series, we appear to have very close to absolute control of the sphenopalatine artery supplied mucosa through an SPA-IMAX ligation approach. From the 3 patients who had severe nosebleeds after surgery and required intervention (surgery and/or anterior-posterior packing), 2 of them had no bleeding from the SPA area previously ligated. The bleeding was exclusively from the anterior ethmoidal area and controlled with specific ligation. Since the source of bleeding was not possible to be identified in one patient who had unilateral SPA-IMAX ligation, this procedure showed at least $98.4 \%$ bleeding control at the SPA supplied mucosa. None of the patients required revisional SPA ligation in our series. These patients should be closely evaluated for the potential for anterior sources of epistaxis prior to SPA-IMAX ligation, so that a concurrent anterior ethmoid artery or greater palatine artery ligation may be considered ${ }^{(13)}$. One potential concern with this procedure is that the vessels were only clipped, not cauterized or sectioned. There may be concern that this could lead to revascularization. However, we have not seen any cases of rebleed from a ligated vessel.

Estimated blood loss was highly variable (161.7 mL \pm 169.4$)$. However, no patients required blood transfusion. Other studies using conventional ligation of the SPA branches have reported the need for blood transfusion ranging from $45-81 \%$ of patients $(9,14)$. It can be argued that SPA-IMAX ligation limits blood loss and perioperative complications such as blood transfusion. In our patient population, there were no immediate perioperative complications requiring additional interventions.

We understand that comprehensive dissection and ligation of all branches of the sphenopalatine artery may have similar results. However, with the ligation of the artery inside the pterygopalatine fossa, all branches are treated including branches that do not pass through the SPA foramen like the pharyngeal branch that passes through the palatovaginal canal and the descending palatine artery decreasing the overall blood flow in the area. In some cases, there are small arterial branches that pass to the nasal cavity through small bone canals other than the SPA foramen. Apparently the SPA/IMAX technique "devascularizes" more arterial branches to the nasal cavity compared to the isolated SPA ligation and avoids the search for different branches due to extensive anatomical variation in this area. Due to limitations in this single arm retrospective case series, we are unable to demonstrate superiority to the standard SPA ligation approach. And it may be argued that the standard SPA ligation approach is technically less challenging. However, in our experience, this procedure can be taught to trainees after a 3-month rotation in rhinology. We believe that for referral centers and experienced surgeons, this combined approach seems to provide a high rate of local control of epistaxis in the sphenopalatine artery supplied mucosa, even in cases of prior failure.

\section{Conclusion}

A dual SPA-IMAX approach seems to provide near complete control over the sphenopalatine supplied region. Especially in anticoagulated patients, a concomitant anterior epistaxis source should be sought.

\section{Authorship contribution}

KP: Data collection and interpretation, manuscript writing/editing; RS: Data collection and interpretation, manuscript writing/ editing; NGL: Manuscript writing/editing. CPN: Principal Investigator and corresponding author, Primary surgeon for patients investigated, Manuscript writing/editing.

\section{Conflict of interest}

We have no conflicts of interest to disclose.

\section{References}

1. Jindal G, Gemmete J, Gandhi D Interventional neuroradiology applications in otolaryngology, head and neck surgery. Otolaryngol Clin North Am. 2012
Dec;45(6):1423-49.

2. Christensen NP, Smith DS, Barnwell SL, Wax MK. Arterial embolization in the management of posterior epistaxis. Otolaryngo Head Neck Surg. 2005 Nov;133(5):748-53.
3. Sacks R, Sacks PL, Chandra R. Chapter 3: Epistaxis. Am J Rhinol Allergy. 2013 MayJun;27 Suppl 1:S9-10.

4. Nouraei SA, Maani T, Hajioff D, Saleh HA Mackay IS. Outcome of endoscopic sphe- 
nopalatine artery occlusion for intractable epistaxis: a 10-year experience. Laryngoscope. 2007 Aug;117(8):1452-6.

5. Simmen DB, Raghavan $U$, Briner $H R$, Manestar M, Groscurth P, Jones NS. The anatomy of the sphenopalatine artery for the endoscopic sinus surgeon. Am J Rhinol. 2006 Sep-Oct;20(5):502-5.

6. Budrovich R, Saetti R. Microscopic and endoscopic ligature of the sphenopalatine artery. Laryngoscope. 1992 Dec;102(12 Pt 1):1391-4.

7. McClurg SW, Carrau R. Endoscopic management of posterior epistaxis: a review. Acta Otorhinolaryngol Ital. 2014 Feb;34(1):1-8

8. Kumar S, Shetty A, Rockey J, Nilssen E. Contemporary surgical treatment of epistaxis. What is the evidence for sphenopalatine artery ligation? Clin Otolaryngol Allied Sci. 2003 Aug;28(4):360-3.

9. Voegels RL, Thomé DC, Iturralde PP, Butugan O. Endoscopic ligature of the sphenopalatine artery for severe posterior epistaxis. Otolaryngol Head Neck Surg. 2001 Apr;124(4):464-7.

10. Abdelkader $M$, Leong SC, White PS Endoscopic control of the sphenopalatine artery for epistaxis: long-term results. J Laryngol Otol. 2007 Aug;121(8):759-62.

11. McDermott AM, O'Cathain E, Carey BW, O'Sullivan P, Sheahan P. Sphenopalatine Artery Ligation for Epistaxis: Factors Influencing Outcome and Impact of Timing of Surgery. Otolaryngol Head Neck Surg. 2016 Mar;154(3):547-52.

12. Felippu A, Mora R, Guastini L. Endoscopic transnasal cauterization of the anterior ethmoidal artery. Acta Otolaryngol. 2011 Oct;131(10):1074-8.

13. Butrymowicz A, Weisstuch A, Zhao A, Agarwal J, Pinheiro-Neto CD. Endoscopic endonasal greater palatine artery cauterization at the incisive foramen for control of anterior epistaxis. Laryngoscope. 2016
May;126(5):1033-8.

14. Barlow DW, Deleyiannis FWB, Pinczower EF. Effectiveness of surgical management of epistaxis at a tertiary care center. Laryngoscope. 1997 Jan;107:21-4.

Carlos D. Pinheiro-Neto MD PhD

Division of Otolaryngology

Head and Neck Surgery

Albany Medical Center

47 New Scotland Avenue, MC-41

Albany, NY, 12208

USA

Tel: +1-518-262 5575

E-mail: pinheic@amc.edu 